Article

\title{
Boundedness of a Class of Oscillatory Singular Integral Operators and Their Commutators with Rough Kernel on Weighted Central Morrey Spaces
}

\author{
Yongliang Zhou ${ }^{1}$, Dunyan Yan ${ }^{1}$ and Mingquan Wei ${ }^{2, *(1)}$ \\ 1 School of Mathematical Sciences, University of Chinese Academy of Sciences, Beijing 100049, China; \\ zhouyongliang19@mails.ucas.ac.cn (Y.Z.); ydunyan@ucas.ac.cn (D.Y.) \\ 2 School of Mathematics and Statistics, Xinyang Normal University, Xinyang 464000, China \\ * Correspondence: weimingquan11@mails.ucas.ac.cn
}

Received: 15 June 2020; Accepted: 28 August 2020; Published: 30 August 2020

check for updates

\begin{abstract}
In this paper, we establish the boundedness of a class of oscillatory singular integral operators with rough kernel on central Morrey spaces. Moreover, the boundedness for each of their commutators on weighted central Morrey spaces was also obtained. We generalized some existing results.
\end{abstract}

Keywords: oscillatory singular integral; commutator; weighted; central Morrey space

\section{Introduction}

Morrey spaces play an significant role in harmonic analysis and partial differential equations. After Morrey [1] introduced that to investigate the local behavior of solutions of the second-order elliptic partial differential equations in 1938, Morrey space was studied by quite a few authors. For example, in the study of the boundedness of Riesz potential on the Morrey space, the first remarkable result was due to Spanne [2] and the second milestone result was due to Adams [3]. Later, the boundedness of Hardy-Littlwood maximal function and singular integral operators on Morrey spaces was obtained by Chiarenza and Frasca [4] in 1987. In addition, for more boundedness of other operators on Morrey space, see [5-7] et al.

Now we review some definitions of Morrey type spaces. Given $1 \leq p<\infty$ and $0 \leq \lambda<n$, the Morrey space $M^{p, \lambda}\left(\mathbb{R}^{n}\right)$ is defined as the set of real-valued measurable function $f$ on $\mathbb{R}^{n}$ enjoying

$$
\|f\|_{M^{p, \lambda}\left(\mathbb{R}^{n}\right)}=\sup _{\left(x_{0}, r\right) \in \mathbb{R}^{n} \times(0, \infty)} r^{-\frac{\lambda}{p}}\left(\int_{B\left(x_{0}, r\right)}|f(x)|^{p} d x\right)^{\frac{1}{p}}<\infty,
$$

where $B\left(x_{0}, r\right)$ is the Euclidean ball with center $x_{0} \in \mathbb{R}^{n}$ and radius $r \in(0, \infty),\left|B\left(x_{0}, r\right)\right| \approx r^{n}$. By slightly modifying the definition, we can get some generalizations of Morrey space, such as

(1) When $r^{-\frac{\lambda}{p}}$ is replaced by $\varphi\left(x_{0}, r\right)^{-1}\left|B\left(x_{0}, r\right)\right|^{-\frac{1}{p}}$, where $\varphi\left(x_{0}, r\right)$ is a positive measurable function on $\mathbb{R}^{n} \times(0, \infty)$, we obtain the generalized Morrey space $M^{p, \varphi}$ and refer to [8,9] for the known results of $M^{p, \varphi}$ for some suitable $\varphi$.

(2) If substituting $\left(\int_{B\left(x_{0}, r\right)}|f(x)|^{p} d x\right)^{\frac{1}{p}}$ with $\|f\|_{L^{p}\left(B\left(x_{0}, r\right) ; \omega\right)}=\left(\int_{B\left(x_{0}, r\right)}|f(x)|^{p} \omega(x) d x\right)^{\frac{1}{p}}$, where $\omega$ is a non-negative locally integrable function, we gain Morrey spaces with weight. As an example, Komori and Shirai [10] defined the weighted Morrey space, which is a natural generalization of classical weighted Lebesgue space. They also showed the boundedness of some classical integral operators and their commutators on weighted Morrey Space. 
In this paper, we focus on local versions of Morrey spaces. More precisely, denoting the ball with center at origin and radius $r$ by $B=B(0, r)=\left\{y \in \mathbb{R}^{n}:|y|<r\right\}$, the central Morrey space $M^{p, \lambda}\left(\mathbb{R}^{n}\right)$ is defined as the set of function $f$ with finite norm $\|f\|_{M^{p, \lambda}\left(\mathbb{R}^{n}\right)}$, where

$$
\|f\|_{M^{p, \lambda}\left(\mathbb{R}^{n}\right)}:=\sup _{r>0} r^{-\frac{\lambda}{p}} \cdot\|f\|_{L^{p}(B)} .
$$

The weighted central Morrey space $M_{\omega}^{p, k}\left(\mathbb{R}^{n}\right)$ is defined as the set of function $f$ with finite norm $\|f\|_{M_{\omega}^{p, k}\left(\mathbb{R}^{n}\right)^{\prime}}$ where

$$
\|f\|_{M_{\omega}^{p, k}\left(\mathbb{R}^{n}\right)}:=\sup _{r>0} \omega(B)^{-\frac{k}{p}} \cdot\|f\|_{L^{p}(B ; \omega)}
$$

where $0<k<1$, $\omega$ be a weight, $\omega(B)=\int_{B} \omega(x) d x$.

Before giving the main results of this paper, let us state some existing results.

Denote the unit sphere in $\mathbb{R}^{n}$ by $\mathbb{S}^{n-1}$. Suppose that $\Omega$ is a homogeneous function with degree zero, has mean value zero on $\mathbb{S}^{n-1}$ and belongs to $L^{q}\left(\mathbb{S}^{n-1}\right)(1<q \leq \infty)$; then the Calderón-Zygmund singular integral with a rough kernel $\Omega$ is defined by

$$
T_{\Omega}(f)(x)=p . v \cdot \int_{\mathbb{R}^{n}} \frac{\Omega(x-y)}{|x-y|^{n}} f(y) d y .
$$

It is worth pointing out that the kernel in the above formula is a convolution kernel. However, there are many kinds of operators which have non-convolution kernels, such as the Fourier transform and Radon transform [11], which belong to oscillatory integrals. Due to F. Ricci and E. M. Stein, the following form of oscillatory singular integrals was studied in [12]:

$$
T_{K} f(x)=p \cdot v \cdot \int_{\mathbb{R}^{n}} e^{i P(x, y)} K(x-y) f(y) d y,
$$

where $P(x, y)$ is a real valued polynomial defined as $\mathbb{R}^{n} \times \mathbb{R}^{n}$, and $K(x)$ is a standard Calderón-Zygmund kernel. That means $K$ satisfies:

(a) $K(x)$ is a $C^{1}$-continuous away from the origin;

(b) $K(x)=\frac{\Omega\left(x^{\prime}\right)}{|x|^{n}}$ with $\Omega$ homogeneous of degree 0 on $\mathbb{S}^{n-1}$;

(c) $\int_{S^{n-1}} \Omega\left(x^{\prime}\right) d \sigma\left(x^{\prime}\right)=0$.

The following theorem is the main result in [12], which was the first result concerning the boundedness of oscillatory sigular integral operator with polynomial phase.

Theorem 1. If $K(x)$ satisfies (a)-(c), then the operator $T_{K}$ can be extended to be a bounded operator on $L^{p}\left(\mathbb{R}^{n}\right)$ to itself, with $1<p<+\infty$, and the norm of this operator depends only on the total degree of $P(x, y)$, but not on the coefficients of $P(x, y)$.

By examining Condition (a), Lu and Zhang [13] introduced new conditions: (d) $\Omega\left(x^{\prime}\right) \in L^{q}\left(\mathbb{S}^{n-1}\right)$, $1<q \leq \infty$ for some $q$. With those mild conditions, they considered a more general oscillatory singular integral operator with rough kernel $T$ which is defined by

$$
T f(x)=p \cdot v \cdot \int_{\mathbb{R}^{n}} e^{i P(x, y)} \frac{\Omega(x-y)}{|x-y|^{n}} f(y) d y,
$$

where $P(x, y)$ is a real valued polynomial defined as $\mathbb{R}^{n} \times \mathbb{R}^{n}$ and $\Omega$ is a rough kernel to be specified later, and promoted Theorem 1 to the following theorem.

Theorem 2. If $\Omega$ satisfies (b)-(d), then the operator $T$ can be extended to be a bounded operator on $L^{p}\left(\mathbb{R}^{n}\right)$ to itself, with $1<p<+\infty$, and the norm of this operator depends only on the total degree of $P(x, y)$, but not on the coefficients of $P(x, y)$. 
In addition, Jiang and Lu [14] gave the weighted form of Theorem 2.

Theorem 3. Suppose $\Omega$ satisfies (b)-(d). Then for any real polynomial $P(x, y), T$ is bounded on $L^{p}(w)$ in each of the following cases:

(A) $q^{\prime} \leq p<\infty(p \neq 1)$ and $w \in A_{p / q^{\prime}}$;

(B) $1<p \leq q(p \neq \infty)$ and $w^{-1 /(p-1)} \in A_{p^{\prime} / q^{\prime}}$, where $1 / p+1 / p^{\prime}=1$ and $1 / q+1 / q^{\prime}=1$.

It should be mentioned that Yu et al. [15] studied the boundedness of singular integral operators and their commutators with rough kernel on weighted central Morrey spaces. For more about the boundedness of operators with rough kernels, readers can refer to [16-21].

For a function $b \in L_{l o c}\left(\mathbb{R}^{n}\right)$, let $A$ be a linear operator on some measurable function space. The commutator between $A$ and $b$ is defined by $[b, A] f(x):=b(x) A f(x)-A(b f)(x)$. The commutator of oscillatory singular integral operators with rough kernel $[b, T]$ is defined by

$$
[b, T] f(x)=p \cdot v \cdot \int_{\mathbb{R}^{n}} e^{i P(x, y)} \frac{\Omega(x-y)}{|x-y|^{n}}(b(x)-b(y)) f(y) d y,
$$

where $T$ is as in (1).

Commutators of oscillatory singular operators have been considered by many authors. The boundedness and weighted boundedness for commutators of oscillatory singular operators were studied by Chen and Zhu [22] and Ding and $\mathrm{Lu}$ [23] respectively, with $\Omega$ being a rough kernel satisfying some conditions.

On the basis of these works, we studied the boundedness of oscillatory singular integral operators with rough kernel and their commutator on center Morrey spaces and weighted center Morrey spaces, respectively.

Now we are in a position to state our main results. Here and in what follows, the operator $T$ and its commutators $[b, T]$ are always defined by (1) and (2), respectively.

Theorem 4. Let $\Omega \in L^{q}\left(\mathbb{S}^{n-1}\right), 1<q \leq \infty, p \in\left(q^{\prime}, \infty\right)$. If the operator $T$ is bounded on $L^{p}\left(\mathbb{R}^{n}\right)$, then $T$ is bounded on $M^{p, \lambda}$.

Theorem 5. Let $\Omega \in L^{q}\left(\mathbb{S}^{n-1}\right), 1<q \leq \infty, p \in\left(q^{\prime}, \infty\right), w \in A_{p / q^{\prime}}$. If $T$ is bounded on $L^{p}(w)$, then $T$ is bounded on $M_{w}^{p, k}$.

Theorem 6. Let $\Omega \in L^{q}\left(\mathbb{S}^{n-1}\right), 1<q \leq \infty, p \in\left(q^{\prime}, \infty\right), b \in B M O\left(\mathbb{R}^{n}\right)$. If the operator $[b, T]$ is bounded on $L^{p}\left(\mathbb{R}^{n}\right)$, then $[b, T]$ is bounded on $M^{p, \lambda}$.

Theorem 7. Let $\Omega \in L^{q}\left(\mathbb{S}^{n-1}\right), 1<q \leq \infty, p \in\left(q^{\prime}, \infty\right), w \in A_{p / q^{\prime}}, b \in B M O\left(\mathbb{R}^{n}\right)$. If $[b, T]$ is bounded on $L^{p}(w)$, then $[b, T]$ is bounded on $M_{w}^{p, k}$.

Remark 1. In the above theorems, the well-definedness of the operators or commutators on weighted Morrey spaces must be checked before the proof. Fortunately, this can proceed in a very similar manner as Remark 7.2 in [24], so we omit the details.

Remark 2. In fact, our theorems says that the (weighted) $L^{p}$ boundedness of $T$ and its commutators $[b, T]$ imply the boundedness on (weighted) Morrey spaces. As a consequence, we can deduce the (weighted) boundedness of $T$ on Morrey spaces if the conditions of Theorem 1, Theorem 2 or Theorem 3 are satisfied.

Remark 3. In [25], Fu et al. gave the boundedness of a class of oscillatory integral operators with standard Calderón-Zygmund kernel and their commutators with symbol in BMO on weighted Morrey spaces. Yu et al. [15] proved the boundedness of singular integral operators with rough kernel and their commutators 
with symbol in $\lambda$-central BMO on the weighted $\lambda$-central Morrey spaces. Our result can be seen generalizations of both results in $[15,25]$ in some sense.

Remark 4. In [26], V. S. Guliyev outlines a very similar results, which are the boundedness of homogeneous singular integrals with rough kernel on the local generalized Morrey spaces $L M_{p, \varphi}^{\left\{x_{0}\right\}}$ for $s^{\prime} \leq p$ or $p<s$ and the commutator operators formed by a local BMO function $b$ and these rough operators on the local generalized Morrey spaces $L M_{p, \varphi}^{\left\{x_{0}\right\}}$. Essentially, Theorems 4 and 6 of our paper are consistent with the main results in [26] in terms of the line of proof, while our arguments are more direct.

Throughout this paper, for a real number $p>1$, we denote $p^{\prime}$ by $1 / p+1 / p^{\prime}=1$. The letter $C$ appearing in this paper is a constant which is independent of the main variables, but may vary from line to line. Denote $t B$ by $B(0, t r)$ for a real number $t>0$. Use symbol $T_{b}$ for the commutator $[b, T]$.

\section{Preliminaries}

Here are some definitions and lemmas needed in the proof of our main results.

In the study of weighted inequalities, an interesting type of weight function is $A_{p}$ weight, which characterizes the weighted $L^{p}$ boundedness of many operators in harmonic analysis, such as the Riesz transforms. Below are the definitions of $A_{p}(1<p<\infty)$ weight and $A_{\infty}$ weight, and the relationship between them.

Definition 1. We say a non-negative function $\omega(x)$ belongs to the Muckenhoupt class $A_{p}$ with $1<p<\infty$ if there exists a constant $C>1$ such that

$$
\left(\frac{1}{|Q|} \int_{Q} \omega(x) d x\right)\left(\frac{1}{|Q|} \int_{Q} \omega(x)^{1-p^{\prime}} d x\right)^{p-1} \leq C
$$

and $[\omega]_{A_{p}}$ denotes the infimum of $C$.

Definition 2. We say $\omega \in A_{\infty}$ if there exist two constant $C$ and $\delta>0$ such that for any measurable set $Q \subset B$,

$$
\frac{\omega(Q)}{\omega(B)} \leq C\left(\frac{|Q|}{|B|}\right)^{\delta}
$$

Lemma 1. ([20]) $A_{\infty}=\bigcup_{1<p<\infty} A_{p}$. If $\omega \in A_{p}$ with $1<p<\infty$, then $\omega \in A_{r}$ for all $r>p$, and $\omega \in A_{q}$ for some $1<q<p$.

On the one hand, the study of BMO function space has its own value. For example, its duality with Hardy space $H^{1}$ has crucial theoretical worth; on the other hand, as a symbolic function for forming commutators, the BMO function also plays an important role in the study of the commutator boundedness. The following is the definition and some properties of the (weighted) BMO function.

Definition 3. A locally integrable function $b$ is said to be in $B M O\left(\mathbb{R}^{n}\right)$ if for any ball $B \subset \mathbb{R}^{n}$

$$
\|b\|_{B M O\left(\mathbb{R}^{n}\right)}=\sup _{B} \frac{1}{|B|} \int_{B}\left|b(x)-b_{B}\right| d x<\infty,
$$

where $b_{B}=\frac{1}{|B|} \int_{B} b(x) d x$.

Lemma 2. ([20]) For all $1<p<\infty$ we have

$$
\|b\|_{B M O\left(\mathbb{R}^{n}\right)} \approx \sup _{B}\left(\frac{1}{|B|} \int_{B}\left|b(x)-b_{B}\right|^{p} d x\right)^{\frac{1}{p}} .
$$


Lemma 3. ([21,27]) Let $1 \leq p<\infty, b \in B M O\left(\mathbb{R}^{n}\right)$. Then for any ball $B \subset \mathbb{R}^{n}$,

$$
\left|b_{2^{\lambda} B}-b_{B}\right| \leq 2^{n} \lambda\|b\|_{B M O\left(\mathbb{R}^{n}\right)} .
$$

Definition 4. A locally integrable function $b$ is said to be in $B M O(\omega)$ if for any ball $B \subset \mathbb{R}^{n}$

$$
\|b\|_{B M O(w)}=\sup _{B} \frac{1}{\omega(B)} \int_{B}\left|b(x)-b_{B, \omega}\right| \omega(x) d x<\infty,
$$

where $b_{B, \omega}=\frac{1}{\omega(B)} \int_{B} b(y) \omega(y) d y$.

Lemma 4. ([28]) Let $1 \leq p<\infty, b \in B M O(w)$. Then there exists a constant $C$ such that

$$
\left(\frac{1}{\omega(B)} \int_{B}\left|b(x)-b_{B, \omega}\right|^{p} \omega(x) d x\right)^{\frac{1}{p}} \leq C\|b\|_{B M O(w)} .
$$

For the sake of conciseness, we will only prove Theorems 6 and 7, since Theorems 4 and 5 are simpler.

\section{Proofs of Main Results}

Compared with the argument in [26], where the author uses the relevant conclusions of the weighted Hardy operator, our method is quite straightforward. With the help of discrete ring decomposition, Holder's inequalities, the relevant properties of the BMO function, some properties of $A_{p}$ weight, etc., we can proof the desired result.

Proof of Theorem 6. As usual, we decompose $f$ as $f=f \chi_{2 B}+f \chi_{(2 B)^{c}}:=f_{1}+f_{2}$. Then

$$
\left\|T_{b} f\right\|_{L^{p}(B)}=\left\|T_{b}\left(f_{1}+f_{2}\right)\right\|_{L^{p}(B)} \leq\left\|T_{b} f_{1}\right\|_{L^{p}(B)}+\left\|T_{b} f_{2}\right\|_{L^{p}(B)},
$$

By the $L^{p}$ boundedness of $T_{b}$, we have

$$
\begin{aligned}
\left\|T_{b} f_{1}\right\|_{L^{p}(B)} & \leq\left\|T_{b} f_{1}\right\|_{L^{p}} \\
& \leq C\left\|f_{1}\right\|_{L^{p}}=C\|f\|_{L^{p}(2 B)} \\
& =C(2 r)^{\frac{\lambda}{p}}(2 r)^{-\frac{\lambda}{p}}\|f\|_{L^{p}(2 B)} \\
& \leq C r^{\frac{\lambda}{p}}\|f\|_{M^{p, \lambda}} .
\end{aligned}
$$

Note that when $x \in B$ and $y \in(2 B)^{c}$,

$$
\begin{aligned}
|y-x| \geq|y|-|x| & >|y|-r \\
& >|y|-\frac{|y|}{2} \\
& =\frac{|y|}{2}>r
\end{aligned}
$$


We have the following estimates

$$
\begin{aligned}
\left|T_{b} f_{2}(x)\right| & =\left|p \cdot v \cdot \int_{\mathbb{R}^{n}} e^{i P(x, y)} \frac{\Omega(x-y)}{|x-y|^{n}}[b(x)-b(y)] f_{2}(y) d y\right| \\
& =\left|\int_{(2 B)^{c}} e^{i P(x, y)} \frac{\Omega(x-y)}{|x-y|^{n}}[b(x)-b(y)] f(y) d y\right| \\
& =\left|\sum_{j=1}^{\infty} \int_{2^{j+1} B \backslash 2^{j} B} e^{i P(x, y)} \frac{\Omega(x-y)}{|x-y|^{n}}[b(x)-b(y)] f(y) d y\right| \\
& \leq \sum_{j=1}^{\infty} \int_{2^{j+1} B \backslash 2^{j} B} \frac{|\Omega(x-y)|}{|x-y|^{n}}|b(x)-b(y)||f(y)| d y \\
& \leq C \sum_{j=1}^{\infty} \int_{2^{j+1} B \backslash 2^{j} B} \frac{|\Omega(x-y)|}{|y|^{n}}|b(x)-b(y)||f(y)| d y \\
& \leq C \sum_{j=1}^{\infty} \frac{1}{\left(2^{j} r\right)^{n}} \int_{2^{j+1} B}|\Omega(x-y)||b(x)-b(y)||f(y)| d y \\
& \leq C\left\{\sum_{j=1}^{\infty} \frac{1}{\left(2^{j} r\right)^{n}} \int_{2^{j+1} B}|\Omega(x-y)|\left|b(x)-b_{2^{j+1} B}\right||f(y)| d y\right. \\
& \left.+\sum_{j=1}^{\infty} \frac{1}{\left(2^{j} r\right)^{n}} \int_{2^{j+1} B}|\Omega(x-y)|\left|b_{2^{j+1} B}-b(y)\right||f(y)| d y\right\} \\
& =: C\left(T_{b} f_{21}(x)+: T_{b} f_{22}(x)\right) .
\end{aligned}
$$

We consider $T_{b} f_{2}(x)$ in two different cases, i.e. $\Omega \in L^{\infty}\left(\mathbb{S}^{n-1}\right)$ and $\Omega \in L^{q}\left(\mathbb{S}^{n-1}\right)(1<q<\infty)$.

Case I. $\Omega \in L^{\infty}\left(\mathbb{S}^{n-1}\right)$.

Using Holder's inequalities, we have

$$
\begin{aligned}
T_{b} f_{21}(x) & \leq\|\Omega\|_{L^{\infty}\left(\mathbb{S}^{n-1}\right)} \sum_{j=1}^{\infty} \frac{1}{\left(2^{j} r\right)^{n}}\left|b(x)-b_{2^{j+1} B}\right| \int_{2^{j+1} B}|f(y)| d y \\
& \leq C \sum_{j=1}^{\infty} \frac{1}{\left(2^{j} r\right)^{n}}\left|b(x)-b_{2^{j+1} B}\right|\|f\|_{L^{p}\left(2^{j+1} B\right)}\left|2^{j+1} B\right|^{\frac{1}{p^{\prime}}} \\
& =C \sum_{j=1}^{\infty}\left|b(x)-b_{2^{j+1} B}\right| \frac{1}{\left(2^{j} r\right)^{n}}\left(2^{j+1} r\right)^{\frac{\lambda}{p}}\left(2^{j+1} r\right)^{-\frac{\lambda}{p}}\|f\|_{L^{p}\left(2^{j+1} B\right)}\left|2^{j+1} B\right|^{\frac{1}{p^{\prime}}} \\
& \leq C\|f\|_{M^{p, \lambda}} \sum_{j=1}^{\infty}\left|b(x)-b_{2^{j+1} B}\right|\left(2^{j+1} r\right)^{\frac{\lambda-n}{p}} .
\end{aligned}
$$

For the term $T_{b} f_{22}(x)$, we have the following estimates

$$
\begin{aligned}
& T_{b} f_{22}(x) \leq\|\Omega\|_{L^{\infty}\left(\mathbb{S}^{n-1}\right)} \sum_{j=1}^{\infty} \frac{1}{\left(2^{j} r\right)^{n}} \int_{2^{j+1} B}\left|b(y)-b_{2^{j+1} B}\right||f(y)| d y \\
\leq & C \sum_{j=1}^{\infty} \frac{1}{\left(2^{j} r\right)^{n}}\left(\int_{2^{j+1} B}\left|b(y)-b_{2^{j+1} B}\right|^{p^{\prime}} d y\right)^{\frac{1}{p^{\prime}}}\left(\int_{2^{j+1} B}|f(y)|^{p} d y\right)^{\frac{1}{p}} \\
= & C \sum_{j=1}^{\infty} \frac{1}{\left(2^{j} r\right)^{n}}\left|2^{j+1} B\right|^{\frac{1}{p^{\prime}}}\left(\frac{1}{\left|2^{j+1} B\right|} \int_{2^{j+1} B}\left|b(y)-b_{2^{j+1} B}\right|^{p^{\prime}} d y\right)^{\frac{1}{p^{\prime}}}\left(2^{j+1} r\right)^{\frac{\lambda}{p}}\left(2^{j+1} r\right)^{-\frac{\lambda}{p}}\|f\|_{L^{p}\left(2^{j+1} B\right)} \\
\leq & C\|f\|_{M^{p, \lambda}}\|b\|_{B M O} \sum_{j=1}^{\infty}\left(2^{j+1} r\right)^{\frac{\lambda-n}{p}} \\
\leq & C\|f\|_{M^{p, \lambda}}\|b\|_{B M O} r^{\frac{\lambda-n}{p}} .
\end{aligned}
$$


Case II. $\Omega \in L^{q}\left(\mathbb{S}^{n-1}\right)(1<q<\infty)$.

By applying Holder's inequalities, we have

$$
\begin{aligned}
& \int_{2^{j+1} B}|\Omega(x-y)|\left|b(x)-b_{2^{j+1} B}\right||f(y)| d y \\
\leq & \left|b(x)-b_{2^{j+1} B}\right|\left(\int_{2^{j+1} B}|\Omega(x-y)|^{q} d y\right)^{\frac{1}{q}}\|f\|_{L^{q^{\prime}}\left(2^{j+1} B\right)} \\
\leq & C\left|b(x)-b_{2^{j+1} B}\right| \mid \Omega\left\|_{L^{q}\left(\mathbb{S}^{n-1}\right)}\left(2^{j+2} r\right)^{\frac{n}{q}}\right\| f \|_{L^{q^{\prime}}\left(2^{j+1} B\right)} \\
\leq & C\left|b(x)-b_{2^{j+1} B}\right|\left(2^{j+2} r\right)^{\frac{n}{q}}\|f\|_{L^{p}\left(2^{j+1} B\right)}\left|2^{j+1} B\right|^{\frac{1}{q^{\prime}}-\frac{1}{p}} \\
= & C\left|b(x)-b_{2^{j+1} B}\right|\left(2^{j+2} r\right)^{n-\frac{n}{p}}\left(2^{j+1} r\right)^{\frac{\lambda}{p}}\left(2^{j+1} r\right)^{-\frac{\lambda}{p}}\|f\|_{L^{p}\left(2^{j+1} B\right)} \\
\leq & C\left|f \|_{M^{p, \lambda}}\right| b(x)-b_{2^{j+1} B} \mid\left(2^{j+1} r\right)^{n-\frac{n}{p}+\frac{\lambda}{p}},
\end{aligned}
$$

which yields

$$
T_{b} f_{21}(x) \leq C\|f\|_{M^{p, \lambda}} \sum_{j=1}^{\infty}\left|b(x)-b_{2^{j+1} B}\right|\left(2^{j+1} r\right)^{\frac{\lambda-n}{p}} .
$$

From the simple estimate

$$
\begin{aligned}
\left\|b-b_{2^{j+1} B}\right\|_{L^{s}\left(2^{j+1} B\right)} & =\left|2^{j+1} B\right|^{\frac{1}{s}}\left(\frac{1}{\left|2^{j+1} B\right|} \int_{2^{j+1} B}\left|b(y)-b_{2^{j+1} B}\right|^{s} d y\right)^{\frac{1}{s}} \\
& \leq C\left|2^{j+1} B\right|^{\frac{1}{s}}\|b\|_{B M O}
\end{aligned}
$$

We obtain that

$$
\begin{aligned}
& \int_{2^{j+1} B}\left|\Omega(x-y)\left\|b(y)-b_{2^{j+1} B}\right\| f(y)\right| d y \\
\leq & \left(\int_{2^{j+1} B}|\Omega(x-y)|^{q} d y\right)^{\frac{1}{q}}\left\|\left(b-b_{2^{j+1} B}\right) f\right\|_{L^{q^{\prime}\left(2^{j+1} B\right)}} \\
\leq & C\|\Omega\|_{L^{q}\left(\mathbb{S}^{n-1}\right)}\left(2^{j+2} r\right)^{\frac{n}{q}}\left\|\left(b-b_{2^{j+1} B}\right) f\right\|_{L^{q^{\prime}}\left(2^{j+1} B\right)} \\
\leq & C\left(2^{j+2} r\right)^{\frac{n}{q}}\|f\|_{L^{p}\left(2^{j+1} B\right)}\left\|b-b_{2^{j+1} B}\right\|_{L^{q^{\prime} \cdot\left(\frac{p}{q^{\prime}}\right)^{\prime}}\left(_{\left(2^{j+1} B\right)}\right.} \\
= & C\left(2^{j+2} r\right)^{\frac{n}{q}}\|f\|_{L^{p}\left(2^{j+1} B\right)}\left\|b-b_{2^{j+1} B}\right\|_{L^{s}\left(2^{j+1} B\right)} \\
\leq & C\left(2^{j+2} r\right)^{\frac{n}{q}}\|f\|_{L^{p}\left(2^{j+1} B\right)}\left|2^{j+1} B\right|^{\frac{1}{s}}\|b\|_{B M O} \\
= & C\left(2^{j+2} r\right)^{\frac{n}{q}}\left(2^{j+1} r\right)^{\frac{\lambda}{p}}\left(2^{j+1} r\right)^{-\frac{\lambda}{p}}\|f\|_{L^{p}\left(2^{j+1} B\right)}\left|2^{j+1} B\right|^{\frac{1}{s}}\|b\|_{B M O} \\
\leq & C\|f\|_{M^{p, \lambda}}\|b\|_{B M O}\left(2^{j+1} r\right)^{n-\frac{n}{p}+\frac{\lambda}{p}},
\end{aligned}
$$

from which it turns out that

$$
T_{b} f_{22}(x) \leq C\|f\|_{M^{p, \lambda}}\|b\|_{B M O} \sum_{j=1}^{\infty}\left(2^{j+1} r\right)^{\frac{\lambda-n}{p}} \leq C\|f\|_{M^{p, \lambda}}\|b\|_{B M O} r^{\frac{\lambda-n}{p}} .
$$


For both cases, we arrive at the same estimates

$$
\begin{gathered}
T_{b} f_{21}(x) \leq C\|f\|_{M^{p, \lambda}} \sum_{j=1}^{\infty}\left|b(x)-b_{2^{j+1} B}\right|\left(2^{j+1} r\right)^{\frac{\lambda-n}{p}} . \\
T_{b} f_{22}(x) \leq C\|f\|_{M^{p, \lambda}}\|b\|_{B M O^{r}} r^{\frac{\lambda-n}{p}} .
\end{gathered}
$$

We proceed to estimate the $L^{p}$ norm of $T_{b} f_{21}$ and $T_{b} f_{22}$ by using (3) and (4).

From the definition of $B M O$, Lemmas 2 and 3 , we deduce that

$$
\begin{aligned}
\left\|b-b_{B}\right\|_{L^{p}(B)} & =\left(\int_{B}\left|b(y)-b_{B}\right|^{p} d y\right)^{\frac{1}{p}} \\
& =|B|^{\frac{1}{p}}\left(\frac{1}{|B|} \int_{B}\left|b(y)-b_{B}\right|^{p} d y\right)^{\frac{1}{p}} \\
& \leq C r^{\frac{n}{p}}\|b\|_{B M O} .
\end{aligned}
$$

and

$$
\left|b_{2 j B}-b_{2^{j+1} B}\right| \leq C\|b\|_{B M O} .
$$

By the estimates (5) and (6), we have the following estimates

$$
\begin{aligned}
& \left\|T_{b} f_{21}\right\|_{L^{p}(B)}=\left(\int_{B}\left|T f_{21}(x)\right|^{p} d x\right)^{\frac{1}{p}} \\
\leq & C\|f\|_{M^{p, \lambda}} \sum_{j=1}^{\infty}\left(\int_{B}\left|b(x)-b_{2^{j+1} B}\right|^{p} d x\right)^{\frac{1}{p}}\left(2^{j+1} r\right)^{\frac{\lambda-n}{p}} \\
= & C\|f\|_{M^{p, \lambda}} \sum_{j=1}^{\infty}\left\|b-b_{2^{j+1} B}\right\|_{L^{p}(B)}\left(2^{j+1} r\right)^{\frac{\lambda-n}{p}} \\
\leq & C\|f\|_{M^{p, \lambda}} \sum_{j=1}^{\infty}\left[\left\|b-b_{B}\right\|_{L^{p}(B)}+\left\|b_{B}-b_{2 B}\right\|_{L^{p}(B)}+\cdots+\left\|b_{2^{j} B}-b_{2^{j+1} B}\right\|_{L^{p}(B)}\right]\left(2^{j+1} r\right)^{\frac{\lambda-n}{p}} \\
\leq & C\|f\|_{M^{p, \lambda}} \sum_{j=1}^{\infty} r^{\frac{n}{p}}\|b\|_{B M O} j\left(2^{j+1} r\right)^{\frac{\lambda-n}{p}} \\
= & C\|f\|_{M^{p, \lambda}}\|b\|_{B M O} \sum_{j=1}^{\infty} r^{\frac{\lambda}{p}} j\left(2^{j+1}\right)^{\frac{\lambda-n}{p}} \\
\leq & C r^{\frac{\lambda}{p}}\|f\|_{M^{p, \lambda}}\|b\|_{B M O} .
\end{aligned}
$$

Obviously, there holds

$$
\begin{aligned}
\left\|T_{b} f_{22}\right\|_{L^{p}(B)} & =\left(\int_{B}\left|T_{b} f_{22}(x)\right|^{p} d x\right)^{\frac{1}{p}} \\
& \leq C r^{\frac{n}{p}}\|f\|_{M^{p, \lambda}}\|b\|_{B M O} r^{\frac{\lambda-n}{p}} \\
& =C r^{\frac{\lambda}{p}}\|f\|_{M^{p, \lambda}}\|b\|_{B M O},
\end{aligned}
$$


which yields

$$
\begin{aligned}
\left\|T_{b} f_{2}\right\|_{L^{p}(B)} & =\left(\int_{B}\left|T_{b} f_{2}(x)\right|^{p} d x\right)^{\frac{1}{p}} \\
& \leq\left(\int_{B}\left[C\left(T_{b} f_{21}(x)+T_{b} f_{22}(x)\right)\right]^{p} d x\right)^{\frac{1}{p}} \\
& \leq C\left(\left\|T_{b} f_{21}\right\|_{L^{p}(B)}+\left\|T_{b} f_{22}\right\|_{L^{p}(B)}\right) \\
& \leq C r^{\frac{\lambda}{p}}\|f\|_{M^{p, \lambda}}\|b\|_{B M O} .
\end{aligned}
$$

Combining the estimate of $\left\|T_{b} f_{1}\right\|_{L^{p}(B)}$ and (8), we get

$$
\begin{aligned}
\left\|T_{b} f\right\|_{L^{p}(B)} & \leq\left\|T_{b} f_{1}\right\|_{L^{p}(B)}+\left\|T_{b} f_{2}\right\|_{L^{p}(B)} \\
& \leq C r^{\frac{\lambda}{p}}\|f\|_{M^{p, \lambda}}+C r^{\frac{\lambda}{p}}\|f\|_{M^{p, \lambda}}\|b\|_{B M O} \\
& \leq C r^{\frac{\lambda}{p}}\|f\|_{M^{p, \lambda}}
\end{aligned}
$$

which means

$$
\left\|T_{b} f\right\|_{M^{p, \lambda}} \leq C\|f\|_{M^{p, \lambda}}
$$

So we complete the proof.

Proof of Theorem 7. The proof of Theorem 7 is a little similar to that of Theorem 6, but there are some differences because of the appearance of weights.

We also decompose $f$ by $f=f \chi_{2 B}+f \chi_{(2 B)^{c}}:=f_{1}+f_{2}$. Then

$$
\left\|T_{b} f\right\|_{L^{p}(B ; \omega)}=\left\|T_{b}\left(f_{1}+f_{2}\right)\right\|_{L^{p}(B ; \omega)} \leq\left\|T_{b} f_{1}\right\|_{L^{p}(B ; \omega)}+\left\|T_{b} f_{2}\right\|_{L^{p}(B ; \omega)},
$$

By the weighted $L^{p}$ boundedness of $T_{b}$, we have

$$
\begin{aligned}
\left\|T_{b} f_{1}\right\|_{L^{p}(B ; \omega)} & \leq\left\|T_{b} f_{1}\right\|_{L^{p}(\omega)} \leq C\left\|f_{1}\right\|_{L^{p}(\omega)} \\
& =C\|f\|_{L^{p}(2 B ; \omega)}=C \omega(2 B)^{\frac{k}{p}} \omega(2 B)^{-\frac{k}{p}}\|f\|_{L^{p}(2 B ; \omega)} \\
& =C \omega(B)^{\frac{k}{p}}\left(\frac{\omega(2 B)}{\omega(B)}\right)^{\frac{k}{p}} \omega(2 B)^{-\frac{k}{p}}\|f\|_{L^{p}(2 B ; \omega)} \\
& \leq C \omega(B)^{\frac{k}{p}}\|f\|_{M_{\omega}^{p, k} .}
\end{aligned}
$$

Through the similar discussion as in Theorem 6, we obtain

$$
\begin{aligned}
\left|T_{b} f_{2}(x)\right| & \leq C \sum_{j=1}^{\infty} \frac{1}{\left(2^{j} r\right)^{n}} \int_{2^{j+1} B}|\Omega(x-y) \| b(x)-b(y)||f(y)| d y \\
& \leq C\left\{\sum_{j=1}^{\infty} \frac{1}{\left(2^{j} r\right)^{n}} \int_{2^{j+1} B}|\Omega(x-y)|\left|b(x)-b_{2^{j+1} B, \omega}\right||f(y)| d y\right. \\
& \left.+\sum_{j=1}^{\infty} \frac{1}{\left(2^{j} r\right)^{n}} \int_{2^{j+1} B}\left|\Omega(x-y) \| b_{2^{j+1} B, \omega}-b(y)\right||f(y)| d y\right\} \\
& =: C\left(T_{b} f_{23}(x)+T_{b} f_{24}(x)\right) .
\end{aligned}
$$

We now divide our discussion into two cases.

Case I. $\Omega \in L^{\infty}\left(\mathbb{S}^{n-1}\right)$. 
For the term $T_{b} f_{23}(x)$, we have the following pointwise estimates:

$$
\begin{aligned}
& T_{b} f_{23}(x) \leq\|\Omega\|_{L^{\infty}\left(\mathbb{S}^{n-1}\right)} \sum_{j=1}^{\infty} \frac{1}{\left(2^{j} r\right)^{n}}\left|b(x)-b_{2^{j+1} B, \omega}\right| \int_{2^{j+1} B}|f(y)| \omega(y)^{\frac{1}{p}} \omega(y)^{-\frac{1}{p}} d y \\
& \leq C \sum_{j=1}^{\infty} \frac{1}{\left(2^{j} r\right)^{n}}\left|b(x)-b_{2^{j+1} B, \omega}\right|\left(\int_{2^{j+1} B}|f(y)|^{p} \omega(y) d y\right)^{\frac{1}{p}}\left(\int_{2^{j+1} B} \omega(y)^{-\frac{p^{\prime}}{p}} d y\right)^{\frac{1}{p^{\prime}}} \\
& =C \sum_{j=1}^{\infty} \frac{1}{\left(2^{j} r\right)^{n}}\left|b(x)-b_{2^{j+1} B, \omega}\right|\|f\|_{L^{p}\left(2^{j+1} B ; \omega\right)}\left|2^{j+1} B\right|^{\frac{1}{p^{\prime}}}\left(\frac{1}{\left|2^{j+1} B\right|} \int_{2^{j+1} B} \omega(y)^{1-p^{\prime}} d y\right)^{\frac{p-1}{p}} \\
& \leq C \sum_{j=1}^{\infty} \frac{1}{\left(2^{j} r\right)^{n}}\left|b(x)-b_{2^{j+1} B, \omega}\right||| f \|_{L^{p}\left(2^{j+1} B ; \omega\right)}\left|2^{j+1} B\right|^{\frac{1}{p^{\prime}}}\left(\frac{1}{\left|2^{j+1} B\right|} \int_{2^{j+1} B} \omega(y) d y\right)^{-\frac{1}{p}} \\
& =C \sum_{j=1}^{\infty} \frac{1}{\left(2^{j} r\right)^{n}}\left|b(x)-b_{2^{j+1} B, \omega}\right| \omega\left(2^{j+1} B\right)^{\frac{k}{p}} \omega\left(2^{j+1} B\right)^{-\frac{k}{p}}\|f\|_{L^{p}\left(2^{j+1} B ; \omega\right)}\left|2^{j+1} B\right| \omega\left(2^{j+1} B\right)^{-\frac{1}{p}} \\
& \leq C\|f\|_{M_{\omega}^{p, k}} \sum_{j=1}^{\infty}\left|b(x)-b_{2^{j+1} B, \omega}\right| \omega\left(2^{j+1} B\right)^{\frac{k-1}{p}} .
\end{aligned}
$$

To get a precise pointwise estimate for $T_{b} f_{24}(x)$, we need the following observation by using Lemma 4

$$
\begin{aligned}
& \left(\int_{2^{j+1} B}\left|b(y)-b_{2^{j+1} B, \omega}\right|^{p^{\prime}} \omega(y)^{-\frac{p^{\prime}}{p}} d y\right)^{\frac{1}{p^{\prime}}} \\
\leq & \left(\left(\int_{2^{j+1} B}\left|b(y)-b_{2^{j+1} B, \omega}\right|^{p^{\prime} \cdot s} \omega(y) d y\right)^{\frac{1}{s}}\left(\int_{2^{j+1} B} \omega(y)^{\left(-\frac{p^{\prime}}{p}-\frac{1}{s}\right) \cdot s^{\prime}} d y\right)^{\frac{1}{s^{\prime}}}\right)^{\frac{1}{p^{\prime}}} \\
= & \omega\left(2^{j+1} B\right)^{\frac{1}{p^{\prime} \cdot s}}\left(\frac{1}{\omega\left(2^{j+1} B\right)} \int_{2^{j+1} B}\left|b(y)-b_{2^{j+1} B, \omega}\right|^{p^{\prime} \cdot s} \omega(y) d y\right)^{\frac{1}{p^{\prime} \cdot s}}\left(\int_{2^{j+1} B} \omega(y)^{1-c^{\prime}} d y\right)^{\frac{c-1}{c}} \\
\leq & C\|b\|_{B M O(\omega)} \omega\left(2^{j+1} B\right)^{\frac{1}{p^{\prime} \cdot s}}\left|2^{j+1} B\right|^{\frac{c-1}{c}}\left(\frac{1}{\left|2^{j+1} B\right|} \int_{2^{j+1} B} \omega(y)^{1-c^{\prime}} d y\right)^{\frac{c-1}{c}} \\
\leq & C\|b\|_{B M O(\omega)} \omega\left(2^{j+1} B\right)^{\frac{1}{p^{\prime} \cdot s}}\left|2^{j+1} B\right|^{\frac{c-1}{c}}\left(\frac{1}{\left|2^{j+1} B\right|} \int_{2^{j+1} B} \omega(y) d y\right)^{-\frac{1}{c}} \\
= & C\|b\|_{B M O(\omega)}\left|2^{j+1} B\right| \omega\left(2^{j+1} B\right)^{\frac{1}{p^{\prime} \cdot s}-\frac{1}{c}} \\
= & C\|b\|_{B M O(\omega)}\left|2^{j+1} B\right| \omega\left(2^{j+1} B\right)^{-\frac{1}{p}},
\end{aligned}
$$

where $c=\left(s^{\prime} p^{\prime}\right)^{\prime}, s>1$ such that $c>p / q^{\prime}$. By using (10), we now estimate $T_{b} f_{24}(x)$ as

$$
\begin{aligned}
T_{b} f_{24}(x) & \leq\|\Omega\|_{L^{\infty}\left(\mathbb{S}^{n-1}\right)} \sum_{j=1}^{\infty} \frac{1}{\left(2^{j} r\right)^{n}} \int_{2^{j+1} B}\left|b(y)-b_{2^{j+1} B, \omega}\right||f(y)| d y \\
& =\|\Omega\|_{L^{\infty}\left(\mathbb{S}^{n-1}\right)} \sum_{j=1}^{\infty} \frac{1}{\left(2^{j} r\right)^{n}} \int_{2^{j+1} B}\left|b(y)-b_{2^{j+1} B, \omega}\right| \omega(y)^{-\frac{1}{p}}|f(y)| \omega(y)^{\frac{1}{p}} d y \\
& \leq C \sum_{j=1}^{\infty} \frac{1}{\left(2^{j} r\right)^{n}}\left(\int_{2^{j+1} B}\left(\left|b(y)-b_{2^{j+1} B, \omega}\right| \omega(y)^{-\frac{1}{p}}\right)^{p^{\prime}} d y\right)^{\frac{1}{p}}\|f\|_{L^{p}\left(2^{j+1} B ; \omega\right)} \\
& \leq C \sum_{j=1}^{\infty} \frac{1}{\left(2^{j} r\right)^{n}}\|b\|_{B M O(\omega)}\left|2^{j+1} B\right| \omega\left(2^{j+1} B\right)^{-\frac{1}{p}}\|f\|_{L^{p}\left(2^{j+1} B ; \omega\right)}
\end{aligned}
$$




$$
\begin{aligned}
& =C \sum_{j=1}^{\infty}\|b\|_{B M O(\omega)} \omega\left(2^{j+1} B\right)^{-\frac{1}{p}} \omega\left(2^{j+1} B\right)^{\frac{k}{p}} \omega\left(2^{j+1} B\right)^{-\frac{k}{p}}\|f\|_{L^{p}\left(2^{j+1} B ; \omega\right)} \\
& \leq C\|f\|_{M_{\omega}^{p, k}\|b\|_{B M O(\omega)} \sum_{j=1}^{\infty} \omega\left(2^{j+1} B\right)^{\frac{k-1}{p}} .}
\end{aligned}
$$

Case II. $\Omega \in L^{q}\left(\mathbb{S}^{n-1}\right)(1<q<\infty)$.

From Definition 1, we have

$$
\begin{aligned}
& \left(\int_{2^{j+1} B}|f(y)|^{q^{\prime}} \omega(y)^{\frac{q^{\prime}}{p}} \omega(y)^{-\frac{q^{\prime}}{p}} d y\right)^{\frac{1}{q^{\prime}}} \\
& \leq\left(\left(\int_{2^{j+1} B}|f(y)|^{p} \omega(y) d y\right)^{\frac{q^{\prime}}{p}}\left(\int_{2^{j+1} B}\left(\omega(y)^{-\frac{q^{\prime}}{p}}\right)^{\left(\frac{p}{q^{\prime}}\right)^{\prime}} d y\right)^{\frac{1}{\left(\frac{p}{q^{\prime}}\right)^{\prime}}}\right)^{\frac{1}{q^{\prime}}} \\
& =\|f\|_{L^{p}\left(2^{j+1} B ; \omega\right)}\left(\int_{2^{j+1} B} \omega(y)^{1-t^{\prime}} d y\right)^{\frac{t-1}{p}}\left(t=p / q^{\prime}\right) \\
& =\|f\|_{L^{p}\left(2^{j+1} B ; \omega\right)}\left|2^{j+1} B\right|^{\frac{t-1}{p}}\left(\frac{1}{\left|2^{j+1} B\right|} \int_{2^{j+1} B} \omega(y)^{1-t^{\prime}} d y\right)^{\frac{t-1}{p}} \\
& \leq C\|f\|_{L^{p}\left(2^{j+1} B ; \omega\right)}\left|2^{j+1} B\right|^{\frac{m-1}{p}}\left(\frac{1}{\left|2^{j+1} B\right|} \int_{2^{j+1} B} \omega(y) d y\right)^{-\frac{1}{p}} \\
& =C\|f\|_{L^{p}\left(2^{j+1} B ; \omega\right)}\left|2^{j+1} B\right|^{\frac{1}{q^{\prime}}} \omega\left(2^{j+1} B\right)^{-\frac{1}{p}} \\
& =C \omega\left(2^{j+1} B\right)^{\frac{k}{p}} \omega\left(2^{j+1} B\right)^{-\frac{k}{p}}\|f\|_{L^{p}\left(2^{j+1} B ; \omega\right)}\left|2^{j+1} B\right|^{\frac{1}{q^{\prime}}} \omega\left(2^{j+1} B\right)^{-\frac{1}{p}} \\
& \leq C\|f\|_{M_{\omega}^{p, k} \omega\left(2^{j+1} B\right)^{\frac{k-1}{p}}\left|2^{j+1} B\right|^{\frac{1}{q^{\prime}}} .}
\end{aligned}
$$

By using (11), we can get a pointwise estimates for $T_{b} f_{23}(x)$,

$$
\begin{aligned}
& T_{b} f_{23}(x)=\sum_{j=1}^{\infty} \frac{1}{\left(2^{j} r\right)^{n}} \int_{2^{j+1} B}|\Omega(x-y)|\left|b(x)-b_{2^{j+1} B, \omega}\right||f(y)| d y \\
& \leq \sum_{j=1}^{\infty} \frac{1}{\left(2^{j} r\right)^{n}}\left|b(x)-b_{2^{j+1} B, \omega}\right|\left(\int_{2^{j+1} B}|\Omega(x-y)|^{q} d y\right)^{\frac{1}{q}}\|f\|_{L^{q^{\prime}\left(2^{j+1} B\right)}} \\
& \leq C \sum_{j=1}^{\infty} \frac{1}{\left(2^{j} r\right)^{n}}\left|b(x)-b_{2^{j+1} B, \omega}\right|\|\Omega\|_{L^{q}\left(\mathbb{S}^{n-1}\right)}\left(2^{j+2} r\right)^{\frac{n}{q}}\|f\|_{L^{q^{\prime}\left(2^{j+1} B\right)}} \\
& =C \sum_{j=1}^{\infty} \frac{1}{\left(2^{j} r\right)^{n}}\left|b(x)-b_{2^{j+1} B, \omega}\right|\left(2^{j+2} r\right)^{\frac{n}{q}}\left(\int_{2^{j+1} B}|f(y)|^{q^{\prime}} \omega(y)^{\frac{q^{\prime}}{p}} \omega(y)^{-\frac{q^{\prime}}{p}} d y\right)^{\frac{1}{q^{\prime}}} \\
& \leq C \sum_{j=1}^{\infty} \frac{1}{\left(2^{j} r\right)^{n}}\left|b(x)-b_{2^{j+1} B, \omega}\right|\left(2^{j+2} r\right)^{\frac{n}{q}}\|f\|_{M_{\omega}^{p, k}} \omega\left(2^{j+1} B\right)^{\frac{k-1}{p}}\left|2^{j+1} B\right|^{\frac{1}{q^{\prime}}} \\
& \leq C\|f\|_{M_{\omega}^{p, k}} \sum_{j=1}^{\infty}\left|b(x)-b_{2^{j+1} B, \omega}\right| \omega\left(2^{j+1} B\right)^{\frac{k-1}{p}} .
\end{aligned}
$$

From the definition of $A_{p}$ weights and Lemma 4, we have

$$
\left(\int_{2^{j+1} B}\left|b(y)-b_{2^{j+1} B, \omega}\right|^{\frac{p q^{\prime}}{p-q^{\prime}}} \omega(y)^{-\frac{q^{\prime}}{p-q^{\prime}}} d y\right)^{\frac{p-q^{\prime}}{p q^{\prime}}}
$$




$$
\begin{aligned}
& =\left(\int_{2^{j+1} B}\left|b(y)-b_{2^{j+1} B, \omega}\right|^{\frac{p q^{\prime}}{p-q^{\prime}}} \omega(y)^{\frac{1}{m}} \omega(y)^{-\frac{q^{\prime}}{p-q^{\prime}}-\frac{1}{m}} d y\right)^{\frac{p-q^{\prime}}{p q^{\prime}}} \\
& \leq\left(\left(\int_{2^{j+1} B}\left|b(y)-b_{2^{j+1} B, \omega}\right|^{\frac{p q^{\prime}}{p-q^{\prime}} \cdot m} \omega(y) d y\right)^{\frac{1}{m}}\left(\int_{2^{j+1} B} \omega(y)^{\left(-\frac{q^{\prime}}{p-q^{\prime}}-\frac{1}{m}\right) \cdot m^{\prime}} d y\right)^{\frac{1}{m^{\prime}}}\right)^{\frac{p-q^{\prime}}{p q^{\prime}}} \\
& =\omega\left(2^{j+1} B\right)^{\frac{1}{m} \cdot \frac{p-q^{\prime}}{p q^{\prime}}}\left(\frac{1}{\omega\left(2^{j+1} B\right)} \int_{2^{j+1} B}\left|b(y)-b_{2^{j+1} B, \omega}\right|^{\frac{p q^{\prime}}{p-q^{\prime}} \cdot m} \omega(y) d y\right)^{\frac{1}{m} \cdot \frac{p-q^{\prime}}{p q^{\prime}}} \\
& \times\left(\int_{2^{j+1} B} \omega(y)^{1-s^{\prime}} d y\right)^{(s-1) \cdot \frac{1}{s-1} \cdot \frac{p-q^{\prime}}{m^{\prime} p q^{\prime}}}\left(s=\left(m^{\prime} t^{\prime}\right)^{\prime}\right) \\
& \leq C\|b\|_{B M O(\omega)} \omega\left(2^{j+1} B\right)^{\frac{p-q^{\prime}}{m p q^{\prime}}}\left(\int_{2^{j+1} B} \omega(y)^{1-s^{\prime}} d y\right)^{(s-1) \cdot \frac{1}{s-1} \cdot \frac{p-q^{\prime}}{m^{\prime} p q^{\prime}}} \\
& =C\|b\|_{B M O(\omega)} \omega\left(2^{j+1} B\right)^{\frac{p-q^{\prime}}{m p q^{\prime}}}\left|2^{j+1} B\right|^{\frac{p-q^{\prime}}{m^{\prime} p q^{\prime}}}\left(\left(\frac{1}{\left|2^{j+1} B\right|} \int_{2^{j+1} B} \omega(y)^{1-s^{\prime}} d y\right)^{(s-1)}\right)^{\frac{1}{s-1} \cdot \frac{p-q^{\prime}}{m^{\prime} p q^{\prime}}} \\
& \leq C\|b\|_{B M O(\omega)} \omega\left(2^{j+1} B\right)^{\frac{p-q^{\prime}}{m p q^{\prime}}}\left|2^{j+1} B\right|^{\frac{p-q^{\prime}}{m^{\prime} p q^{\prime}}}\left(\frac{1}{\left|2^{j+1} B\right|} \int_{2^{j+1} B} \omega(y) d y\right)^{-\frac{1}{s-1} \cdot \frac{p-q^{\prime}}{m^{\prime} p q^{\prime}}} \\
& =C\|b\|_{B M O(\omega)} \omega\left(2^{j+1} B\right)^{\frac{p-q^{\prime}}{m p q^{\prime}}}-\frac{1}{s-1} \cdot \frac{p-q^{\prime}}{m^{\prime} p q^{\prime}}\left|2^{j+1} B\right|^{\frac{p-q^{\prime}}{m^{\prime} p q^{\prime}}}+\frac{1}{s-1} \cdot \frac{p-q^{\prime}}{m^{\prime} p q^{\prime}} \\
& =C\|b\|_{B M O(\omega)} \omega\left(2^{j+1} B\right)^{-\frac{1}{p}}\left|2^{j+1} B\right|^{\frac{1}{q^{\prime}}} \text {, }
\end{aligned}
$$

where $m$ is some number greater than 1 such that $w \in A_{\left(m^{\prime} t^{\prime}\right)^{\prime}, t}=p / q^{\prime}$. From this

$$
\begin{aligned}
& \left(\int_{2^{j+1} B}\left|b(y)-b_{2^{j+1} B, \omega}\right|^{q^{\prime}}|f(y)|^{q^{\prime}} d y\right)^{\frac{1}{q^{\prime}}} \\
= & \left(\int_{2^{j+1} B}\left|b(y)-b_{2^{j+1} B, \omega}\right|^{q^{\prime}} \omega(y)^{-\frac{q^{\prime}}{p}}|f(y)|^{q^{\prime}} \omega(y)^{\frac{q^{\prime}}{p}} d y\right)^{\frac{1}{q^{\prime}}} \\
\leq & \left(\left(\int_{2^{j+1} B}\left|b(y)-b_{2^{j+1} B, \omega}\right|^{q^{\prime} \cdot\left(\frac{p}{q^{\prime}}\right)^{\prime}} \omega(y)^{\left(-\frac{q^{\prime}}{p}\right) \cdot\left(\frac{p}{q^{\prime}}\right)^{\prime}} d y\right)^{\frac{1}{\left(\frac{p}{q^{\prime}}\right)^{\prime}}}\left(\int_{2^{j+1} B}|f(y)|^{p} \omega(y) d y\right)^{\frac{q^{\prime}}{p}}\right)^{\frac{1}{q^{\prime}}} \\
= & \left(\int_{2^{j+1} B}\left|b(y)-b_{2^{j+1} B, \omega}\right|^{\frac{p q^{\prime}}{p-q^{\prime}}} \omega(y)^{-\frac{q^{\prime}}{p-q^{\prime}}} d y\right)^{\frac{p-q^{\prime}}{p q^{\prime}}}\|f\|_{L^{p}\left(2^{j+1} B ; \omega\right)} \\
\leq & C\|b\|_{B M O(\omega)} \omega\left(2^{j+1} B\right)^{-\frac{1}{p}}\left|2^{j+1} B\right|^{\frac{1}{q^{\prime}}}\|f\|_{L^{p}\left(2^{j+1} B ; \omega\right)} .
\end{aligned}
$$

Taking (13) into consideration, we obtain

$$
\begin{aligned}
T_{b} f_{24}(x) & =\sum_{j=1}^{\infty} \frac{1}{\left(2^{j} r\right)^{n}} \int_{2^{j+1} B}\left|\Omega(x-y) \| b(y)-b_{2^{j+1} B, \omega}\right||f(y)| d y \\
& \leq \sum_{j=1}^{\infty} \frac{1}{\left(2^{j} r\right)^{n}}\left(\int_{2^{j+1} B}|\Omega(x-y)|^{q} d y\right)^{\frac{1}{q}}\left(\left.\int_{2^{j+1} B}\left|b(y)-b_{2^{j+1} B, \omega}\right|\right|^{\prime}|f(y)|^{q^{\prime}} d y\right)^{\frac{1}{q^{\prime}}} \\
& \leq C \sum_{j=1}^{\infty} \frac{1}{\left(2^{j} r\right)^{n}}\|\Omega\|_{L^{q}\left(\mathbb{S}^{n-1}\right)}\left(2^{j+2} r\right)^{\frac{n}{q}}\left(\int_{2^{j+1} B}\left|b(y)-b_{2^{j+1} B, \omega}\right| q^{q^{\prime}}|f(y)|^{q^{\prime}} d y\right)^{\frac{1}{q^{\prime}}}
\end{aligned}
$$




$$
\begin{aligned}
& \leq C \sum_{j=1}^{\infty} \frac{1}{\left(2^{j} r\right)^{n}}\left(2^{j+2} r\right)^{\frac{n}{q}}\|b\|_{B M O(\omega)} \omega\left(2^{j+1} B\right)^{-\frac{1}{p}}\left|2^{j+1} B\right|^{\frac{1}{q^{\gamma}}}\|f\|_{L^{p}\left(2^{j+1} B ; \omega\right)} \\
& =C\|b\|_{B M O(\omega)} \sum_{j=1}^{\infty} \omega\left(2^{j+1} B\right)^{\frac{k-1}{p}} \omega\left(2^{j+1} B\right)^{-\frac{k}{p}}\|f\|_{L^{p}\left(2^{j+1} B ; \omega\right)} \\
& \leq C\|b\|_{B M O(\omega)}\|f\|_{M_{\omega}^{p, k}} \sum_{j=1}^{\infty} \omega\left(2^{j+1} B\right)^{\frac{k-1}{p}} .
\end{aligned}
$$

For both cases, we arrive at the same estimates

$$
\begin{gathered}
T_{b} f_{23}(x) \leq C\|f\|_{M_{\omega}^{p, k}} \sum_{j=1}^{\infty}\left|b(x)-b_{2^{j+1} B, \omega}\right| \omega\left(2^{j+1} B\right)^{\frac{k-1}{p}} . \\
T_{b} f_{24}(x) \leq C\|b\|_{B M O(\omega)}\|f\|_{M_{\omega}^{p, k} \sum_{j=1}^{\infty} \omega\left(2^{j+1} B\right)^{\frac{k-1}{p}} .}
\end{gathered}
$$

In the following, we will proceed to estimate the $L^{p}$ norm of $T_{b} f_{23}$ and $T_{b} f_{24}$ by using (14) and (15). From Definition 4 and Lemma 4, we get

$$
\begin{aligned}
\left\|b-b_{B, \omega}\right\|_{L^{p}(B ; \omega)} & =\left(\int_{B}\left|b(y)-b_{B, \omega}\right|^{p} \omega(y) d y\right)^{\frac{1}{p}} \\
& =\omega(B)^{\frac{1}{p}}\left(\frac{1}{\omega(B)} \int_{B}\left|b(y)-b_{B, \omega}\right|^{p} \omega(y) d y\right)^{\frac{1}{p}} \\
& \leq C \omega(B)^{\frac{1}{p}}\|b\|_{B M O(\omega)} .
\end{aligned}
$$

and

$$
\begin{aligned}
\left|b_{2^{j} B, \omega}-b_{2^{j+1} B, \omega}\right| & =\left|\frac{1}{\omega\left(2^{j} B\right)} \int_{2^{j} B} b(y) \omega(y) d y-b_{2^{j+1} B, \omega}\right| \\
& =\left|\frac{1}{\omega\left(2^{j} B\right)} \int_{2^{j} B}\left(b(y)-b_{2^{j+1} B, \omega}\right) \omega(y) d y\right| \\
& \leq \frac{1}{\omega\left(2^{j} B\right)} \int_{2^{j} B}\left|b(y)-b_{2^{j+1} B, \omega}\right| \omega(y) d y \\
& \leq C \frac{1}{\omega\left(2^{j+1} B\right)} \int_{2^{j+1} B}\left|b(y)-b_{2^{j+1} B, \omega}\right| \omega(y) d y \\
& \leq C\|b\|_{B M O(\omega)}
\end{aligned}
$$

which yields

$$
\left\|b_{2^{j} B, \omega}-b_{2^{j+1} B, \omega}\right\|_{L^{p}(B ; \omega)} \leq C \omega(B)^{\frac{1}{p}}\|b\|_{B M O(\omega)} .
$$

By using the estimate of $\left\|b-b_{B, \omega}\right\|_{L^{p}(B ; \omega)},(16)$ and Lemma 4 , we have

$$
\begin{aligned}
& \left\|T_{b} f_{23}\right\|_{L^{p}(B ; \omega)}=\left(\int_{B}\left|T f_{23}(x)\right|^{p} \omega(x) d x\right)^{\frac{1}{p}} \\
\leq & C\|f\|_{M_{\omega}^{p, k}} \sum_{j=1}^{\infty}\left\|b-b_{2^{j+1} B, \omega}\right\|_{L^{p}(B ; \omega)} \omega\left(2^{j+1} B\right)^{\frac{k-1}{p}} \\
\leq & C\|f\|_{M_{\omega}^{p, k}} \sum_{j=1}^{\infty}\left[\left\|b-b_{B, \omega}\right\|_{L^{p}(B ; \omega)}+\cdots+\left\|b_{2^{j} B, \omega}-b_{2^{j+1} B, \omega}\right\|_{L^{p}(B ; \omega)}\right] \omega\left(2^{j+1} B\right)^{\frac{k-1}{p}}
\end{aligned}
$$




$$
\begin{aligned}
& \leq C\|f\|_{M_{\omega}^{p, k}} \sum_{j=1}^{\infty}\|b\|_{B M O(\omega)^{-}} \omega(B)^{\frac{1}{p}} j \omega\left(2^{j+1} B\right)^{\frac{k-1}{p}} \\
& =C\|f\|_{M_{\omega}^{p, k}}\|b\|_{B M O(\omega)} \omega(B)^{\frac{k}{p}} \sum_{j=1}^{\infty} j\left(\frac{\omega(B)}{\omega\left(2^{j+1} B\right)}\right)^{\frac{1-k}{p}} \\
& \leq C\|f\|_{M_{\omega}^{p, k}}\|b\|_{B M O(\omega)} \omega(B)^{\frac{k}{p}} .
\end{aligned}
$$

From Definition 2 and Lemma 1, we have

$$
\begin{aligned}
& \left\|T_{b} f_{24}\right\|_{L^{p}(B ; \omega)}=\left(\int_{B}\left|T_{b} f_{24}(x)\right|^{p} \omega(x) d x\right)^{\frac{1}{p}} \\
& \leq C \omega(B)^{\frac{1}{p}}\|b\|_{B M O(\omega)}\|f\|_{M_{\omega}^{p, k}} \sum_{j=1}^{\infty} \omega\left(2^{j+1} B\right)^{\frac{k-1}{p}} \\
& =C\|f\|_{M_{\omega}^{p, k}}\|b\|_{B M O(\omega)} \omega(B)^{\frac{k}{p}} \sum_{j=1}^{\infty}\left(\frac{\omega(B)}{\omega\left(2^{j+1} B\right)}\right)^{\frac{1-k}{p}}
\end{aligned}
$$

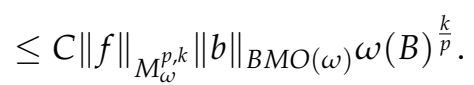

Inserting (17) and (18) into our estimates, we get

$$
\begin{aligned}
\left\|T_{b} f_{2}\right\|_{L^{p}(B ; \omega)} & =\left(\int_{B}\left|T_{b} f_{2}(x)\right|^{p} \omega(x) d x\right)^{\frac{1}{p}} \\
& \leq\left(\int_{B}\left[C\left(T_{b} f_{23}(x)+T_{b} f_{24}(x)\right)\right]^{p} \omega(x) d x\right)^{\frac{1}{p}} \\
& \leq C\left(\left\|T_{b} f_{23}\right\|_{L^{p}(B ; \omega)}+\left\|T_{b} f_{24}\right\|_{L^{p}(B ; \omega)}\right) \\
& \leq C\|f\|_{M_{\omega}^{p, k}}\|b\|_{B M O(\omega)} \omega(B)^{\frac{k}{p}}
\end{aligned}
$$

Combining (9) and (19), it shows

$$
\begin{aligned}
\left\|T_{b} f\right\|_{L^{p}(B ; \omega)} & \leq\left\|T_{b} f_{1}\right\|_{L^{p}(B ; \omega)}+\left\|T_{b} f_{2}\right\|_{L^{p}(B ; \omega)} \\
& \leq C \omega(B)^{\frac{k}{p}}\|f\|_{M_{\omega}^{p, k}}+C\|f\|_{M_{\omega}^{p, k}}\|b\|_{B M O(\omega)} \omega(B)^{\frac{k}{p}} \\
& \leq C \omega(B)^{\frac{k}{p}}\|f\|_{M_{\omega}^{p, k}}
\end{aligned}
$$

which yields

$$
\left\|T_{b} f\right\|_{M_{\omega}^{p, k}} \leq C\|f\|_{M_{\omega}^{p, k}}
$$

We complete the proof.

\section{Conclusions}

We obtain the boundedness of a class of oscillatory singular integral operators with rough kernel and their commutators on the (weighted) central Morrey spaces. In fact, for a large class of operators $T$ which satisfies

$$
|T f(x)| \leq C \int \frac{|\Omega(x-y)|}{|x-y|^{n}}|f(y)| d y \leq C \int \frac{|\Omega(x-y)|}{|y|^{n}}|f(y)| d y
$$

when $x \notin \operatorname{suppf}$, its boundedness on the (weighted) local Morrey space can be obtained as long as it is $L^{p}\left(L^{p}(\omega)\right)$ bounded. The corresponding conclusion also holds for the commutator operators formed by a $B M O$ function $b$ and such operators. 
Author Contributions: Formal analysis, M.W.; writing-original draft preparation, Y.Z.; writing-review and editing, M.W.; supervision, D.Y. All authors have read and agreed to the published version of the manuscript.

Funding: This work was funded by the NNSF of China (grant number 11871452) and the Nanhu Scholar Program for Young Scholars of Xinyang Normal University and Project of Henan Provincial Department of Education (grant number 18A110028).

Conflicts of Interest: All of authors in this article declare no conflict of interest. All of funders in this article support the article's publication.

\section{References}

1. Morrey, C.B. On the solutions of quasi-linear elliptic partial differential equations. Trans. Am. Math. Soc. 1938, 43, 126-166. [CrossRef]

2. Peetre, J . On the theory of $M_{p, \lambda}$. J. Funct. Anal. 1969, 4, 71-87.

3. Adams, D.R. A note on Riesz potentials. Duke Math. J. 1975, 42, 765-778. [CrossRef]

4. Chiarenza, F.; Frasca, M. Morrey spaces and Hardy-Littlewood maximal function. Rend.-Lincei-Mat. Appl. 1987, 7, 273-279.

5. Burenkov, V.I.; Jain, P.; Tararykova, T.V. On boundedness of the Hardy operator in Morrey-type spaces. Math. J. 2012, 2, 52-80.

6. Liu, Y.; Chen, D. The boundedness of maximal Bochner-Riesz operator and maximal commutator on Morrey type spaces. Anal. Theory Appl. 2008, 24, 321-329. [CrossRef]

7. He, Q ; Yan, D. Bilinear fractional integral operators on Morrey spaces. Positivity 2020. [CrossRef]

8. Mizuhara, T. Commutators of singular integral operators on Morrey spaces with general growth functions. In Harmonic Analysis and Nonlinear Partial Differential Equations ; Sūrikaisekikenkyūsho Kōkyūroku: Kyoto, Japan, 1999; Volume 1102, pp. 49-63.

9. Komori, Y.; Mizuhara, T. Factorization of functions in $H^{1}\left(\mathbb{R}^{n}\right)$ and generalized Morrey spaces. Math. Nachrichten 2006, 279, 619-624. [CrossRef]

10. Komori, Y.; Shirai, S. Weighted Morrey spaces and a singular integral operator. Math. Nachrichten 2009, 282, 219-231. [CrossRef]

11. Phong, D.H.; Stein, E.M. Singular integrals related to the Radon transform and boundary value problems. Proc. Natl. Acad. Sci. USA 1984, 80, 7697-7701. [CrossRef]

12. Ricci, F.; Stein, E.M. Harmonic analysis on nilpotent groups and singular integrals I. Oscillatory integrals. J. Funct. Anal. 1987, 73, 179-194. [CrossRef]

13. Lu, S.; Zhang, Y. Criterion on $L^{p}$-boundedness for a class of oscillatory singular integrals with rough kernels. Rev. Mat. Iberoam. 1992, 8, 115-134.

14. Jiang, Y.; Lu, S. Oscillatory singular integrals with rough kernel. J. Beijing Norm. Univ. 1995, 27, 135-145.

15. Yu, X.; Zhang, H.; Zhao, G. Weighted boundedness of some integral operators on weighted $\lambda$-central Morrey space. J. Appl. Math. Univ. Engl. Ed. (Ser. B) 2016, 31, 331-342. [CrossRef]

16. Hu, G. $L^{p}\left(\mathbb{R}^{n}\right)$ boundedness for the commutator of a homogeneous singular integral operator. Stud. Math. 2003, 154, 13-27. [CrossRef]

17. Grafakos, L.; Stefanov, A. $L^{p}$ Bounds for singular integrals and maximal singular integrals with rough kernels. Indiana Univ. Math. J. 1998, 47, 455-469. [CrossRef]

18. Chen, Y.; Ding, Y. $L^{p}$ bounds for the commutators of singular integrals and maximal singular integrals with rough kernels. Trans. Am. Math. Soc. 2014, 367, 1585-1608. [CrossRef]

19. Lu, S.; Ding, Y.; Yan, D. Singular Integral and Related Topics; World Scientific Publishing: Singapore, 2011.

20. Grafakos, L. Classical and Modern Fourier Analysis; Pearson Education, Inc.: Upper Saddle River, NJ, USA, 2004.

21. Lu, S. Four Lectures on Real $H_{p}$ Spaces; World Scientific Publishing: Singapore, 1995.

22. Chen, Y.; Zhu, K. $L^{p}$ Bounds for the commutators of oscillatory singular integrals with rough kernels. Abstr. Appl. Anal. 2014, 2014, 393147. [CrossRef]

23. Ding, Y.; Lu, S. Weighted $L^{p}$-boundedness for higher order commutators of oscillatory singular integrals. Tohoku Math. J. 1996, 48, 437-449. [CrossRef]

24. Shi, M.; Arai, R.; Nakai, E. Commutators of integral operators with functions in Campanato spaces on Orlicz-Morrey spaces. arXiv 2020, arXiv:2007.00468v1. 
25. Fu, Z.; Shi, S.; Lu, S. Boundedness of oscillatory integral operators and their commutators on weighted Morrey spaces. Sci. Sin. 2013, 43, 147-158.

26. Guliyev, V.S. Local generalized Morrey spaces and singular integrals with rough kernel. Azerbaijan J. Math. 2013, 3, 79-94.

27. Torchinsky, A. Real-Variable Methods in Harmonic Analysis; Academic Press: Cambridge, MA, USA, 1986.

28. Muckenhoupt, B.; Wheeden, R. Weighted bounded mean oscillation and Hilbert transform. Stud. Math. 1976, 54, 221-237. [CrossRef]

(C) 2020 by the authors. Licensee MDPI, Basel, Switzerland. This article is an open access article distributed under the terms and conditions of the Creative Commons Attribution (CC BY) license (http:/ / creativecommons.org/licenses/by/4.0/). 